\title{
Properties for certain subclasses of analytic functions with nonzero coefficients
}

Hyo Jeong Lee ${ }^{1}$, Nak Eun Cho ${ }^{* *}$ and Shigeyoshi Owa²

"Correspondence:

necho@pknu.ac.kr

${ }^{1}$ Department of Applied

Mathematics, Pukyong National

University, Busan, 608-737, Korea

Full list of author information is

available at the end of the article

\begin{abstract}
In the present paper, we obtain some mapping and inclusion properties for subclasses of analytic functions by using a linear operator defined by the Gaussian hypergeometric function.
\end{abstract}

MSC: $30 C 45$

Keywords: univalent function; uniformly starlike function; uniformly convex function; Gaussian hypergeometric function; Hadamard product

\section{Introduction}

Let $\mathcal{A}$ denote the class of functions of the form

$$
f(z)=z+\sum_{n=2}^{\infty} a_{n} z^{n} \quad\left(a_{n} \neq 0\right)
$$

which are analytic in the open unit disk $\mathbb{U}=\{z \in \mathbb{C}:|z|<1\}$. We also denote by $\mathcal{S}$ the class of all functions in $\mathcal{A}$ which are univalent in $\mathbb{U}$.

A function $f \in \mathcal{A}$ is said to be in the class $\Re^{t}(A, B, \alpha)$ if

$$
\left|\frac{f^{\prime}(z)-1}{t(A-B)-B\left(f^{\prime}(z)-1\right)}\right|<\alpha \quad(z \in \mathbb{U})
$$

where $A$ and $B$ are complex numbers with $A \neq B, t \in \mathbb{C} \backslash\{0\}$, and $\alpha$ is a positive real number.

In particular, for some real numbers $A$ and $B$ with $-1 \leq B<A \leq 1$ and $\alpha=1$ without any restriction of the coefficients $a_{n}(n \in \mathbb{N}=\{1,2, \ldots\})$ the class $\Re^{t}(A, B, \alpha)$ was introduced by Dixit and Pal [1]. Moreover, by giving specific values $t, A, B$, and $\alpha$ in (1.2), we obtain subclasses studied by various researchers in earlier works (see [2-6]).

A function $f \in \mathcal{A}$ is said to be in the class $\mathcal{U S T}(\alpha)$ if

$$
\Re\left\{\frac{f(z)-f(\xi)}{(z-\xi) f^{\prime}(z)}\right\}>\alpha \quad(z \times \xi \in \mathbb{U} \times \mathbb{U} ; 0 \leq \alpha<1) .
$$

Furthermore, a function $f \in \mathcal{A}$ is said to be in the class $\mathcal{U C V}(\alpha)$ if

$$
\Re\left\{1+\frac{(z-\xi) f^{\prime \prime}(z)}{f^{\prime}(z)}\right\}>\alpha \quad(z \times \xi \in \mathbb{U} \times \mathbb{U} ; 0 \leq \alpha<1) .
$$

๑2014 Lee et al.; licensee Springer. This is an Open Access article distributed under the terms of the Creative Commons Attribution License (http://creativecommons.org/licenses/by/2.0), which permits unrestricted use, distribution, and reproduction in any medium, provided the original work is properly cited. 
The classes $\mathcal{U S T}(0) \equiv \mathcal{U S T}$ and $\mathcal{U C V}(0) \equiv \mathcal{U C V}$ are introduced by Goodman $[7,8]$ (they are called the classes of uniformly starlike and uniformly convex functions, respectively). The classes of uniformly starlike and uniformly convex functions have been extensively studied by Ma and Minda [9] and Rønning [10].

Let us consider the Gaussian hypergeometric function $F(a, b ; c ; z)$ defined by

$$
F(a, b ; c ; z)=\sum_{n=0}^{\infty} \frac{(a)_{n}(b)_{n}}{(c)_{n}} z^{n} \quad(a, b, c \in \mathbb{C} ; c \neq 0,-1,-2, \ldots ; z \in \mathbb{U})
$$

where $(v)_{n}$ is the Pochhammer symbol (or the shifted factorial) defined (in terms of the gamma function) by

$$
\begin{aligned}
(v)_{n}: & =\frac{\Gamma(v+n)}{\Gamma(v)} \\
& = \begin{cases}1 & \text { if } n=0 \text { and } v \in \mathbb{C} \backslash\{0\}, \\
v(v+1) \cdots(v+n-1) & \text { if } n \in \mathbb{N} \text { and } v \in \mathbb{C} .\end{cases}
\end{aligned}
$$

We note that $F(a, b ; c ; z)=F(b, a ; c ; z)$ and

$$
F(a, b ; c ; 1)=\frac{\Gamma(c-a-b) \Gamma(c)}{\Gamma(c-a) \Gamma(c-b)} \quad(\Re\{c-a-b\}>0) .
$$

We also recall (see $[11,12])$ that the function $F(a, b ; c ; z)$ is bounded if $\Re\{c-a-b\}>0$, and it has a pole at $z=1$ if $\Re\{c-a-b\} \leq 0$. Moreover, univalence, starlikeness and convexity properties of $z F(a, b ; c ; z)$ have been extensively studied by Ponnusamy and Vuorinen [13] and Ruscheweyh and Singh [14].

For $f \in \mathcal{A}$, we define the operator $I_{a, b ; c} f$ by

$$
I_{a, b ; c} f(z)=z F(a, b ; c ; z) * f(z)
$$

where $*$ denotes the usual Hadamard product (or convolution) of power series. For a special case of the operator $I_{a, b ; f} f$, we can refer to the paper by Swaminathan [15] and the references cited therein.

In this paper, we obtain a necessary condition for the class $\Re^{t}(A, B, \alpha)$ and sufficient conditions for the classes $\Re^{t}(A, B, \alpha), \mathcal{U S T}(\alpha)$, and $\mathcal{U C V}(\alpha)$, respectively. Moreover, we find a condition for univalency of the operator $I_{a, b ;} f$ defined by (1.3).

\section{Main result}

Theorem 1 Let a function $f$ of the form (1.1) be in the class $\Re^{t}(A, B, \alpha)$ with $a_{n}=\left|a_{n}\right| e^{i \frac{(3 n+1) \pi}{2}}$ $(n \in \mathbb{N} \backslash\{1\})$, then

$$
\sum_{n=2}^{\infty} n(1-\alpha|B|)\left|a_{n}\right| \leq \alpha|t||A-B| .
$$

Proof From the definition of $\Re^{t}(A, B, \alpha)$, we have

$$
\left|f^{\prime}(z)-1\right|<\alpha\left|t(A-B)-B\left(f^{\prime}(z)-1\right)\right| \quad(z \in \mathbb{U}),
$$


and so

$$
\left|\sum_{n=2}^{\infty} n a_{n} z^{n-1}\right|<\alpha\left|t(A-B)-B \sum_{n=2}^{\infty} n a_{n} z^{n-1}\right| .
$$

If we take $z=r e^{i \frac{\pi}{2}}$, then we see that

$$
a_{n} z^{n-1}=\left|a_{n}\right| r^{n-1} \quad(0 \leq r<1) .
$$

Then, by using (2.3) to (2.2), we have

$$
\begin{aligned}
\sum_{n=2}^{\infty} n\left|a_{n}\right| r^{n-1} & <\alpha\left|t(A-B)-B \sum_{n=2}^{\infty} n\right| a_{n}\left|r^{n-1}\right| \\
& <\alpha|t(A-B)|+\alpha|B| \sum_{n=2}^{\infty} n\left|a_{n}\right| r^{n-1}
\end{aligned}
$$

or, equivalently,

$$
\sum_{n=2}^{\infty} n(1-\alpha|B|)\left|a_{n}\right| r^{n-1}<\alpha|t||A-B| .
$$

Letting $r \rightarrow 1^{-}$in (2.4), we have the inequality (2.1). Thus we complete the proof of Theorem 1.

Theorem 2 Let a function $f$ of the form (1.1) be in the class $\mathcal{A}$. If

$$
\sum_{n=2}^{\infty} n(1+\alpha|B|)\left|a_{n}\right| \leq \alpha|t||A-B|,
$$

where $A$ and $B$ are complex numbers with $A \neq B, t \in \mathbb{C} \backslash\{0\}$, and $\alpha$ is a positive real number, then $f \in \mathfrak{R}^{t}(A, B, \alpha)$. The result is sharp for the function defined by

$$
\begin{aligned}
& f(z)=z+\sum_{n=2}^{\infty} \frac{\alpha t(A-B) \epsilon}{n^{2}(n-1)(1+\alpha|B|)} z^{n} \\
& \quad(A, B \in \mathbb{C} ; A \neq B ; t \in \mathbb{C} \backslash\{0\} ;|\epsilon|=1 ; z \in \mathbb{U}) .
\end{aligned}
$$

Proof In view of the definition of $\Re^{t}(A, B, \alpha)$, it suffices to prove that

$$
\left|f^{\prime}(z)-1\right|<\alpha\left|t(A-B)-B\left(f^{\prime}(z)-1\right)\right| \quad(z \in \mathbb{U})
$$

From (2.6), we have

$$
\left|\sum_{n=2}^{\infty} n a_{n} z^{n-1}\right|<\alpha\left|t(A-B)-B \sum_{n=2}^{\infty} n a_{n} z^{n-1}\right| .
$$


Hence it is sufficient to show that

$$
\sum_{n=2}^{\infty} n\left|a_{n}\right| r^{n-1}<\alpha\left(|t||A-B|-|B| \sum_{n=2}^{\infty} n\left|a_{n}\right| r^{n-1}\right)
$$

which is equivalent to the relation

$$
\sum_{n=2}^{\infty} n(1+\alpha|B|)\left|a_{n}\right| r^{n-1}<\alpha|t||A-B|
$$

Letting $r \rightarrow 1^{-}$in (2.7), we have

$$
\sum_{n=2}^{\infty} n(1+\alpha|B|)\left|a_{n}\right| \leq \alpha|t||A-B|
$$

Therefore, by the assumption (2.5), we prove that $f \in \Re^{t}(A, B, \alpha)$.

Theorem 3 Let a function $f$ of the form (1.1) be in the class $\mathcal{A}$. If

$$
\sum_{n=2}^{\infty}((3-\alpha) n-2)\left|a_{n}\right| \leq 1-\alpha \quad(0 \leq \alpha<1)
$$

then $f \in \mathcal{U S T}(\alpha)$. The result is sharp for the function

$$
f(z)=z+\sum_{n=2}^{\infty} \frac{(1-\alpha) \epsilon}{n(n-1)((3-\alpha) n-2)} z^{n} \quad(0 \leq \alpha<1 ;|\epsilon|=1) .
$$

Proof It suffices to show that

$$
\left|\frac{f(z)-f(\xi)}{(z-\xi) f^{\prime}(z)}-1\right| \leq 1-\alpha \quad(0 \leq \alpha<1 ;(z, \xi) \in \mathbb{U} \times \mathbb{U}) .
$$

We have

$$
\begin{aligned}
& \left|\frac{f(z)-f(\xi)}{(z-\xi) f^{\prime}(z)}-1\right| \\
& \quad=\left|\frac{\sum_{n=2}^{\infty} a_{n}\left(\xi^{n-1}+z \xi^{n-2}+\cdots+z^{n-1}\right)-\sum_{n=2}^{\infty} n a_{n} z^{n-1}}{1+\sum_{n=2}^{\infty} n a_{n} z^{n-1}}\right| \\
& \quad \leq \frac{\sum_{n=2}^{\infty} 2(n-1)\left|a_{n}\right|}{1-\sum_{n=2}^{\infty} n\left|a_{n}\right|}
\end{aligned}
$$

which is bounded by $1-\alpha$ if the assumption (2.8) is satisfied. Thus we complete the proof of Theorem 3.

Theorem 4 Let a function $f$ of the form (1.1) be in the class $\mathcal{A}$. If

$$
\sum_{n=2}^{\infty} n(2 n-1-\alpha)\left|a_{n}\right| \leq 1-\alpha \quad(0 \leq \alpha<1)
$$


then $f \in \mathcal{U C V}(\alpha)$. The result is sharp for the function

$$
f(z)=z+\sum_{n=2}^{\infty} \frac{(1-\alpha) \epsilon}{n^{2}(n-1)(2 n-1-\alpha)} z^{n} \quad(0 \leq \alpha<1 ;|\epsilon|=1 ; z \in \mathbb{U}) .
$$

Proof It suffices to show that

$$
\left|\frac{(z-\varphi) f^{\prime \prime}(z)}{f^{\prime}(z)}\right|<1-\alpha \quad(0 \leq \alpha<1 ;(z, \varphi) \in \mathbb{U} \times \mathbb{U}) .
$$

We have

$$
\left|\frac{(z-\varphi) f^{\prime \prime}(z)}{f^{\prime}(z)}\right|=\left|\frac{(z-\varphi) \sum_{n=2}^{\infty} n(n-1) a_{n} z^{n-2}}{1-\sum_{n=2}^{\infty} n a_{n} z^{n-1}}\right| \leq \frac{2 \sum_{n=1}^{\infty} n(n-1)\left|a_{n}\right|}{1-\sum_{n=2}^{\infty} n\left|a_{n}\right|},
$$

which is bounded by $1-\alpha$ if the assumption (2.9) is satisfied. Thus we complete the proof of Theorem 4 .

Theorem 5 Let $a, b \in \mathbb{C} \backslash\{0\}$ and $c>|a|+|b|$. If $f \in \mathfrak{R}^{t}(A, B, \alpha)$ with $a_{n}=\left|a_{n}\right| e^{i \frac{(3 n+1) \pi}{2}}$, $0<|B|<1$, and

$$
\frac{\Gamma(c-|a|-|b|) \Gamma(c)}{\Gamma(c-|a|) \Gamma(c-|b|)} \leq \frac{1-\alpha|B|}{1+\alpha|B|}+1,
$$

then $I_{a, b ; f} \in \Re^{t}(A, B, \alpha)$, where the operator $I_{a, b ; c} f$ is defined by (1.3).

Proof We want to prove from Theorem 2 that

$$
T_{1}:=\sum_{n=2}^{\infty} n(1+\alpha|B|)\left|A_{n}\right| \leq \alpha|t||A-B|,
$$

where

$$
A_{n}=\frac{(a)_{n-1}(b)_{n-1}}{(c)_{n-1}(1)_{n-1}} a_{n} .
$$

Since, from Theorem 1,

$$
\begin{aligned}
\left|a_{n}\right| & \leq \frac{\alpha|t||A-B|}{n(1-\alpha|B|)} \\
T_{1} & \leq \frac{\alpha|t||A-B|(1+\alpha|B|)}{1-\alpha|B|} \sum_{n=2}^{\infty} \frac{(|a|)_{n-1}(|b|)_{n-1}}{(c)_{n-1}(1)_{n-1}} \\
& =\frac{\alpha|t||A-B|(1+\alpha|B|)}{1-\alpha|B|}\left(\sum_{n=0}^{\infty} \frac{(|a|)_{n}(|b|)_{n}}{(c)_{n}(1)_{n}}-1\right) \\
& =\frac{\alpha|t||A-B|(1+\alpha|B|)}{1-\alpha|B|}\left(\frac{\Gamma(c-|a|-|b|) \Gamma(c)}{\Gamma(c-|a|) \Gamma(c-|b|)}-1\right) \\
& \leq \alpha|t||A-B|,
\end{aligned}
$$

which completes the proof of Theorem 5 . 
We introduce the following lemma which is needed for the proof of the next theorem.

Lemma 1 [16] Let $\omega$ be regular in the unit disk $\mathbb{U}$ with $\omega(0)=0$. Then, if $|\omega(z)|$ attains a maximum value on the circle $|z|=r(0 \leq r<1)$ at a point $z_{0}$, we can write

$$
z_{0} \omega^{\prime}\left(z_{0}\right)=k \omega\left(z_{0}\right) \quad(k \geq 1)
$$

Theorem 6 Let a function $f$ of the form (1.1) be in the class $\mathcal{A}$. Assume

$$
\left|\frac{\left(I_{a, b ; c} f(z)\right)^{\prime}-1}{1-\alpha}\right|^{\beta}\left|\frac{z\left(I_{a, b ; f} f(z)\right)^{\prime \prime}}{\left(I_{a, b ; f} f(z)\right)^{\prime}-\alpha}\right|^{\gamma}<\frac{1}{2^{\gamma}} \quad(z \in \mathbb{U})
$$

for some real $\alpha(0 \leq \alpha<1), \beta>0$, and $\gamma>0$. Then

$$
\left|\left(I_{a, b ; f} f(z)\right)^{\prime}-1\right|<1-\alpha \quad(z \in \mathbb{U}) .
$$

Proof Let us define $\omega$ by

$$
\omega(z)=\frac{\left(I_{a, b ;} f(z)\right)^{\prime}-1}{1-\alpha} \quad(z \in \mathbb{U}) .
$$

Then it follows that $\omega$ is analytic in $\mathbb{U}$ with $\omega(0)=0$. By (2.10), we have

$$
\begin{aligned}
|\omega(z)|^{\beta}\left|\frac{z \omega^{\prime}(z)}{\omega(z)+1}\right|^{\gamma} & =|\omega(z)|^{\beta+\gamma}\left|\frac{z \omega^{\prime}(z)}{\omega(z)} \frac{1}{\omega(z)+1}\right|^{\gamma} \\
& <\frac{1}{2^{\gamma}} \quad(z \in \mathbb{U}) .
\end{aligned}
$$

Suppose that there exists a point $z_{0} \in \mathbb{U}$ such that

$$
\max _{|z| \leq\left|z_{0}\right|}|\omega(z)|=\left|\omega\left(z_{0}\right)\right|=1
$$

Then, by Lemma 1, we can put

$$
\frac{z_{0} \omega^{\prime}\left(z_{0}\right)}{\omega\left(z_{0}\right)}=k \geq 1
$$

Hence, we obtain

$$
\begin{aligned}
\left|\omega\left(z_{0}\right)\right|^{\beta}\left|\frac{z_{0} \omega^{\prime}\left(z_{0}\right)}{\omega\left(z_{0}\right)+1}\right|^{\gamma} & =\left|\frac{z_{0} \omega^{\prime}\left(z_{0}\right)}{\omega\left(z_{0}\right)+1}\right|^{\gamma} \\
& \geq\left(\frac{k}{2}\right)^{\gamma} \geq \frac{1}{2^{\gamma}},
\end{aligned}
$$

which contradicts the condition (2.12). This shows that

$$
|\omega(z)|=\left|\frac{\left(I_{a, b ; f} f(z)\right)^{\prime}-1}{1-\alpha}\right|<1 \quad(z \in \mathbb{U}) .
$$

Thus we complete the proof of Theorem 6 . 
Remark 1 The condition (2.11) in Theorem 6 implies that

$$
\Re\left\{\left(I_{a, b ; f} f(z)\right)^{\prime}\right\}>0 \quad(z \in \mathbb{U}) .
$$

Therefore, by the Noshiro-Warschawski theorem [17], the operator $I_{a, b ; f} f$ is univalent in $\mathbb{U}$ under the restrictions of Theorem 6.

\section{Competing interests}

The authors declare that they have no competing interests.

\section{Authors' contributions}

All authors jointly worked on the results and they read and approved the final manuscript.

\section{Author details}

${ }^{1}$ Department of Applied Mathematics, Pukyong National University, Busan, 608-737, Korea. ${ }^{2}$ Department of Mathematics, Faculty of Education, Yamato University, Katayama 2-5-1, Suita, Osaka 564-0082, Japan.

\section{Acknowledgements}

The authors would like to express their thanks to the referees for many valuable advices regarding a previous version of this paper. This research was supported for the second author by the Basic Science Research Program through the National Research Foundation of Korea (NRF) funded by the Ministry of Education, Science and Technology (No. 2011-0007037).

\section{Received: 18 July 2014 Accepted: 2 December 2014 Published: 12 Dec 2014}

\section{References}

1. Dixit, KK, Pal, SK: On a class of univalent functions related to complex order. Indian J. Pure Appl. Math. 26(9), 889-896 (1995)

2. Caplinger, TR, Causey, WM: A class of univalent functions. Proc. Am. Math. Soc. 39, 357-361 (1973)

3. Kim, JA, Cho, NE: Properties of convolutions for hypergeometric series with univalent functions. Adv. Differ. Equ. 2013, $101(2013)$

4. Dashrath: On some classes related to spiral-like univalent and multivalent functions. PhD thesis, Kanpur University, Kanpur (1984)

5. Padmanabhan, KS: On a certain class of functions whose derivatives have a positive real part in the unit disc. Ann. Pol. Math. 23, 73-81 (1970/1971)

6. Ponnusamy, S, Rønning, F: Starlikeness properties for convolutions involving hypergeometric series. Ann. Univ. Mariae Curie-Skłodowska, Sect. A 52, 141-155 (1998)

7. Goodman, AW: On uniformly convex functions. Ann. Pol. Math. 56, $87-92$ (1991)

8. Goodman, AW: On uniformly starlike functions. J. Math. Anal. Appl. 155, 364-370 (1991)

9. Ma, W, Minda, D: Uniformly convex functions. Ann. Pol. Math. 57, 166-175 (1992)

10. Rønning, F: Uniformly convex functions and a corresponding class of starlike functions. Proc. Am. Math. Soc. 118 190-196 (1993)

11. Owa, S, Srivastava, HM: Univalent and starlike generalized hypergeometric functions. Can. J. Math. 39, 1057-1077 (1987)

12. Whitteaker, ET, Watson, GN: A Course of Modern Analysis: An Introduction to the General Theory of Infinite Processes and Analytic Functions: With an Account of the Principal Transcendental Functions, 4th edn. Cambridge University Press, Cambridge (1927)

13. Ponnusamy, S, Vuorinen, M: Univalence and convexity properties for Gaussian hypergeometric functions. Preprint 82 Department of Mathematics, University of Helsinki (1995)

14. Ruscheweyh, S, Singh, V: On the order of starlikeness of hypergeometric functions. J. Math. Anal. Appl. 113, 1-11 (1986)

15. Swaminathan, A: Certain sufficiency conditions on Gaussian hypergeometric functions. J. Inequal. Pure Appl. Math. 5 Article $83(2004)$

16. Jack, IS: Functions starlike and convex of order $\alpha$. J. Lond. Math. Soc. 3, 469-474 (1971)

17. Duren, PL: Univalent Functions. Springer, New York (1983)

10.1186/1029-242X-2014-506

Cite this article as: Lee et al.: Properties for certain subclasses of analytic functions with nonzero coefficients. Journal of Inequalities and Applications 2014, 2014:506 